\title{
Vaccination against tuberculosis
}

\section{Vacinação contra a tuberculose}

In 1908 two researchers, Albert Calmette and Camille Guerin found that Mycobacterium bovis became less virulent when it was grown on a medium containing beef bile. For 13 years they re-cultured the bacteria every three weeks until 231 generations later they had a strain that was mild enough to pose no danger to humans, and could produce immunity against tuberculosis without causing the disease. And so in 1921 BCG was first used to vaccinate children against tuberculosis in France. In the UK a trial sponsored by the Medical Research Council in 1950 showed that BCG provides substantial protection against tuberculosis, ${ }_{1}$ following which the vaccine came to be widely adopted first in Europe and then globally. But while credited with helping end the tuberculosis epidemic in Europe, the vaccine has been less successful in the developing world. 2 The reasons for this have been much debated. ${ }^{3}$ A consensus is forming around the view that while neonatal vaccination can provide protection against childhood manifestation of the disease the immunity wanes over time. A recent report adds credence to this view. In 979 child household contacts (median age seven years) of 414 adult patients who had sputum smear positive pulmonary tuberculosis $770(79 \%)$ children had a BCG scar. Three of them were diagnosed to have active tuberculosis compared with 10 out of 209 unvaccinated children. Thus presence of a BCG scar was associated with a relative risk reduction of $92 \%$ for tuberculosis in this cohort. 4 Repeated BCG vaccination or BCG vaccination in individuals already sensitised by environmental mycobacteria is ineffective. In Brazil school children in two cities were enrolled in a study of efficacy of BCG revaccination. The crude incidence of tuberculosis was 29.3 per 100000 person years of follow up in the revaccinated group compared to 30.2 per 100000 person years in the control group. 5 The memory response may be rendered ineffective by repeated exposure to mycobacteria whether from revaccination or environmental exposure, or both. This could be a reason why the widespread use of BCG has not significantly affected the growing pandemic of human tuberculosis. As things stand, we now know that BCG protects against serious paediatric forms of tuberculosis, and in some parts of the world against adult pulmonary tuberculosis. But populations in high-burden countries are not benefited by the current regimen. BCG revaccination of previously vaccinated individuals offers little apparent benefit. 6

BCG being a live vaccine will require a period of replication in the host to generate optimal immune response which can be inhibited by residual immunity from earlier vaccinations. That being the case would antigen(s) rather than live bacteria succeed in boosting a previously established immune response?

A number of candidate antigens have been cloned and expressed as recombinant proteins in Escherichia coli both for developing sero-diagnostic tests ${ }^{7}$ and as constituents of novel vaccines. ${ }^{8} \mathrm{~A}$ vaccine comprising of two antigenic proteins, ESAT-6 (early secretory antigenic target 6) and AG85 B (tuberculosis antigen 85B) as a fusion protein intended to serve as a booster vaccine for individuals previously vaccinated with BCG is in Phase I trial. 9 Choice of adjuvant and vehicle to carry the antigen can significantly influence the immune response. The adjuvant used in the case of ESAT-6 and AG85B fusion protein is MPL-A (monophosphoryl phospholipid) derived from the cell wall of a Gram negative bacteria with proven immuno-stimulatory action, but other formulations are also under development.10,11 Vaccines containing protein antigens with appropriate adjuvants have been shown to function effectively in animal studies.

M. tuberculosis is an intracellular pathogen for which the defence relies on cell-mediated immunity, and T-cell effector mechanisms rather than antibody are needed to control or eliminate the bacteria. Virus vectors carrying genes for the appropriate antigens can not only deliver them within a recipient cell but also many of them have the added advantage of generating strong cell-mediated immune responses themselves. Recombinant modified vaccinia virus Ankara expressing tuberculosis antigen $85 \mathrm{~A}$ is proven to be safe. The virus does not replicate in humans and dies after expressing its proteins. Antigen 85A is present in all mycobacterial species being an enzyme involved in building the cell wall of the bacteria. Fowl pox is another candidate vector. Such vaccines should not be affected by prior mycobacterial sensitization of the host, and are potentially usable as booster vaccines if proven to be effective. A vaccine MVA-85A has just gone through a phase I trial in UK and clinical trial in the Gambia.

Assuming that the results are encouraging, then one future strategy in the control of tuberculosis could be primary vaccination with BCG followed by booster vaccine at a later date using MVA-85A or similar vaccine. Such a prime-boost strategy is based on using two separate vaccines, each of which includes the same antigen 
but in different forms. Many questions still remain unanswered. BCG works effectively but only for 10-15 years. Why does immunity wane? What is the long term evolution of the immune response to BCG vaccination? We know that $\mathrm{CD}_{4} \mathrm{~T}$ cells are among the most important in the protective response against Mycobacterium tuberculosis, because in HIV infection loss of $\mathrm{CD}_{4}+\mathrm{T}$ cells greatly increase susceptibility to both acute and reactivation tuberculosis. $\mathrm{CD}_{4}+\mathrm{T}$ cells produce interferon- $\gamma$ and several cytokines including interleukin-2, both of which have important role in fighting against tuberculosis. 12 Studies in animal models have provided such insights and helped our understanding of the immune response to tuberculosis in human disease. The mouse is relatively more resistant and the guinea pig more sensitive to M. tuberculosis and thus provide clinical pictures of slow and rapid progression respectively of the disease in humans.

During the process of attenuation over thirteen years it is plausible that BCG lost genes necessary for longer lasting protection. BCG lacks the potent secreted T-cell antigens ESAT-6 (6-kilo Dalton early secretory antigen target) and CFP-10 (10-kilo Dalton culture filtrate protein) as a result of deletions. Both are present in all virulent mycobacteria studied.13 Recombinant BCG containing genes absent or under expressed in current BCG strains provides enhanced protection in animal models. Two live recombinant BCG vaccines are currently undergoing trials. If trial results are successful, and depending upon the evolution of the immune response recombinant $\mathrm{BCG}$ may come to replace old BCG in the prime-boost strategy.

As new vaccines become available, and several candidates are in different stages of trials 14 two challenges remain to be addressed. What are the correlates of immunity and how should they be measured, particularly in the large majority of the world's population carrying latent infection? Containment of latent infection requires on-going immune response and tools for measuring it need to be developed. Secondly, M. tuberculosis is known to alter its gene expression radically during the course of infection. What effect does it have on the host's immune response, and in such cases would the new vaccines continue to remain protective?

\section{G.J.Ebrahim}

Department of International Child Health. Institute of Child Health. University of London

Editorial advisor

\section{Referências}

1. Hart PD'A, Sutherlnd I. BCG and vole bacillus vaccines in the prevention of tuberculosis in adolescence and early adult life: final report to the Medical Research Council. BMJ. 1977; 2: 293-5.

2. Tuberculosis Prevention Trial, Madras. Trial of BCG vaccines in south India for tuberculosis prevention. Indian J Med Res. 1980; 72 (Suppl):1-74.

3. Smith D, Wiegeshaus E, Balasubramanian V. An analysis of some hypotheses related to the Chingleput Bacille Calmette-Guerine trial. Clin Infect Dis. 2000; 31 (Suppl 3): S77-80.

4. Soysal A, Millinton KA, Babir M, Dosanjh D, Aslan Y, Deeks JJ, Efe S, Staveley I, Ewer K, Lalvani A. Effect of BCG vaccination on risk of Mycobacterium tuberculosis in children with household tuberculosis contact: a prospective community based study. Lancet. 2005; 366: 1443-51.

5. Rodrigues LC, Pereira SM, Cunha SS, Genser B, Ichihara MY, de Brito SC, Hijjar MA, Dourado I, Cruz AA, Sant'Anna C, Bierrenbach AL, Barreto ML. Effect of BCG revaccination on incidence of tuberculosis in school-aged children in Brazil: the BCGREVAC cluster-randomised trial. Lancet. 2005; 366: 1290-5.

6. Leung CC, Tam CM, Chan SL, Chang-Yeung M, Chan CK, Chang KC. Efficacy of BCG revaccination programme in a cohort given BCG vaccination at birth in Hong Kong. Int J Tuberc Lung Dis. 2001; 5: 717-23.

7. Weldingh K, Rosenkrands I, Okkels LM, Doherty TM, Andersen P. Assessing the serodiagnostic potential of 35 Mycobacterium tuberculosis proteins and identification of four novel serological antigens. J Clin Microbiol. 2005; 43: 57-65.

8. Weinrich OA, van Pinxteren LA, Meng Okkels L, Birk RP, Anderson P. Protection of mice with a tuberculosis subunit vaccine based on a fusion protein of Antigen 85B and ESAT-6. Infect Immun. 2001; 69: 2773-8.

9. Doherty TM, Rook G. Progress and hindrances in tuberculosis vaccine development. Lancet. 2006; 367: 947-9.

10. de Fonseca DP, Frerichs J, Singh M, Snippe H, Verheul AF. Induction of antibody and T-cell responses by immunization with ISCOMS containing the 38-kilo Dalton protein of Mycobacterium tuberculosis. Vaccine. 2000; 19: 122-31.

11. Lima KM, Bonato VL, Faccioli LH, Brandão IT, dos Santos SA, Coelho-Castelo AA. Comparison of different delivery systems of vaccination for the induction of protection against tuberculosis in mice. Vaccine. 2001; 19: 3518-25.

12. Flynn JL, Chan J. Immunology of tuberculosis. Ann Rev Immunol. 2001; 19: 93-129.

13. Pym AS, Brodin P, Majlessi L, Brosh R, Demangel C, Williams A, Griffiths KE, Marchal G, Leclerc C, Colet ST. Recombinant BCG exporting ESAT-6 confers enhanced protection against tuberculosis. Nat Med. 2003; 9: 533-9.

14. Orme IM. Current progress in tuberculosis vaccine development. Vaccine. 2005; 23: 105-8 